\title{
Recherches sur la toxicité et la valeur trypanopréventive du moranylate d'éthidium
}

\section{Note de présentation}

\author{
par R. SAUVEL ef M. THOMÉ
}

L'histoire des complexes à base de Moranyl (ou suramine dans les pays de langue anglaise), débuta en 1942, lorsque LOURIE observa que le mélange d'une solution de suramine et de diamidines donnait naissance à un sel insoluble, présentant des propriétés pharmacologiques différentes de celles de ses constituants.

GUIMARAES ef LOURIE (1951), SCHNEIDER et MONTEZIN (1954), COSAR et Collaborateurs (1954). BEAUDIMENT et Collaborateurs (1954). montrèrent par la suite que chez les animaux de laboratoire et chez l'homme, ce sel possède une toxicité inférieure et des propriétés préventives supérieures à celles des diamidines employées seules.

WILLIAMSON ET DESOWITZ (1956) eurent alors l'idée de former des complexes analogues avec les trypanocides utilisés dans les trypanosomiases animales : bromure d'Ethidium, Antrycidc, Bérénil qui, comme les diamidines, possèdent des propriétés basiques. Les premiers essais réalisés par ces auteurs les amenèrent à la conclusion que c'est le complexe d'Ethidium qui possède les propriétés préventives les plus marquées, mais que, malheureusement, les réactions provoquées par son administration sont trop importantes ef le rendent pratiquement inutilisable.

Néanmoins très intéressés par le remarquable pouvoir préventif de ce complexe, nous avons voulu reprendre son étude pour voir si les conclusions de nos confrères anglais s'appliquaient à nos zones d'expérimentation ainsi qu'aux animaux dont nous disposions.

Reçu pour publication : février 1961

Rev. Elev. Méd. vét. Pays trop., 1961, 14, no 2.
C'est ainsi que nous avons fait entreprendre plusieurs séries d'expériences qui ont débuté en février 1957 et qui ont été réalisées à Farcha, Fort-Lamy (République du Tchad), à Bouar (République Centrafricaine) et à Riggil et Sulfa (République du Cameroun).

Au départ, tant à Bouar qu'à Farcha, les études ont porté sur les propriétés physiques, la toxicité locale et la toxicité générale du moranylate d'Ethidium. Nous nous sommes intéressés ensuite très rapidement aux qualités préventives de ce produit.

Les résultats satisfaisants obtenus à Riggil et à Bovar nous ont amenés à entreprendre une expérimentation d'une plus grande envergure dans les conditions de brousse à Sulfa. Cette expérience prouva la valeur trypanopréventive certaine du moranylate d'Ethidium, mais montra également que, lorsque la surveillance et les précautions n'étaient plus aussi strictes que celles du laboratoire, les réactions locales représentaient un obstacle à la généralisation de l'emploi du moranylate d'Ethidium.

Considérant que la valeur préventive du produit méritait une étude plus poussée, nous avons décidé de faire reprendre l'étude de la toxicité du moranylate d'Ethidium en l'utilisant sous des formes et avec des excipients différents et en l'introduisant dans l'organisme par diverses voies.

Ces expériences ont été menées à Bouar et à Farcha mais plus particulièrement à Farcha.

Ces différentes expérimentations dont nous venons de parler sont exposées dans des notes à part publiées sous les noms de ceux qui furent chargés de ces expérimentations.

Pour avoir une vue générale de ces études qui ont porté sur quatre années, nous avons pensé 
qu'il était préférable d'en résumer les résultats dans cette note de présentation.

\section{A. - Mode et forme d'administration}

Les essais entrepris par voie sous-cutanée, intra-musculaire, intra-veineuse en utilisant le moranylate d'Ethidium en solution aqueuse, avec addition de gel d'alumine, en excipient huileux (avec ou sans cortisone), avec de la lanoline (avec ou sans cortisone) ou sous forme d'implants plus ou moins délitables (avec ou sans cortisone) permettent de conclure que c'est en excipient huileux ou, mieux, sous formes d'implants introduits en sous-cutanée dans le fanon que le moranylate d'Ethidium provoque les réactions locales les plus faibles. Quant à la dose il semble qu'elle ne doive pas dépasser $5 \mathrm{mg} / \mathrm{kg}$ pour éviter tout phénomène de toxicité générale.

\section{B. - Pouvoir trypanopréventif}

En région de galeries forestières où les conditions d'infestation naturelle sont très dures ef interdisent tout élevage (infestation au bout de 14 à 52 jours selon l'époque de l'année), le moranylate d'Ethidium a conféré aux animaux traités une protection qui n'a pas été inférieure à six mois et qui a pu atteindre un an.

En région sahélienne type «steppe » où l'infestation trypanosomienne est moyenne les animaux traités ont été protégés plus d'une année et même deux ans. 